functional and cognitive decline. The phenomenology of $\mathrm{AD}$ delusions has not been adequately addressed; neither has whether AD delusions relate to specific cognitive deficits.

Conclusions: Delusions in AD are common and are related to greater rates of cognitive decline. Future studies that explore the relationship between $\mathrm{AD}$, delusions and cognitive decline need to control for methodological issues and need to examine the phenomenology of delusions and their relationship with specific cognitive deficits.

\section{Are affective prosodic deficits evident in bipolar disorder?}

\section{A O'Regan, SL Rossell, NR Joshua}

Mental Health Research Institute of Victoria, Melbourne, Australia

Background: Deficits in affective prosodic processing have been well established in patients with schizophrenia. Yet, no study has examined this skill in a bipolar sample, this was the aim of the current study.

Methods: Three matched groups (patients with bipolar disorder, patients with schizophrenia and healthy controls) completed four affective prosody tasks from the Comprehensive Affective Testing System (CATS); emotional prosody discrimination (EPD), name emotional prosody (NEP), attend to prosody during conflicting prosody (CPP) and attend to meaning during conflicting prosody (CPM).

Results: No significant group effects were found for any of the four tasks on either correct reaction time (RT) or accuracy measures. A task effect, however, was found across all four tasks showing a similar pattern for the RT (correct) and accuracy data; superior performance on EPD, then CPP, followed by NEP then CPM.

Conclusions: The lack of group differences for each of the four tasks may be a reflection of the CATS paradigms lacking the capacity to effectively discriminate subtle differences in performance. An extensive literature indicates that individuals with schizophrenia and bipolar disorder do tend to perform worse on a range of affect tasks that use fearful and sad stimuli. Therefore, the lack of significant group effects in the current tasks may be because of an insufficient number of stimuli per affective category used in each task, thus poor power; alternatively, incongruent accent must be considered, that is, CATS uses American not Australian actors. Further study is recommended using experimental measures of affective prosody.

\section{Omega-3 fatty acids and mood disorders}

\author{
C Owen ${ }^{1,2}$, A-M Rees ${ }^{1}$, G Parker ${ }^{1}$ \\ 'The Black Dog Institute, Sydney, Australia; and 2University of New South Wales
}

Background: Over the past century, consumption of omega-3 polyunsaturated fatty acids has decreased dramatically in Western diets, while consumption of omega- 6 has increased. There is mounting evidence suggesting that deficits in dietary intake of omega-3 are associated with a range of health problems, including mood disorders. This review will outline the epidemiological and clinical evidence for an association between omega-3 depletion and mood disorders, including unipolar, bipolar and perinatal depression and suicide risk.

Method: Relevant published studies are reviewed and theories regarding the mechanisms of action are presented.

Results: Strong correlations between rates of seafood consumption and mood disorders have been observed in several epidemiological studies. Biochemical studies indicate that omega-3 levels are lower in the tissue of people with depressive disorders than matched controls. The results of several randomized controlled trials indicate that omega-3 supplementation may have therapeutic benefit for depression suffers, although not all trials have shown a benefit.

Conclusions: Deficits in omega- 3 fatty acids are associated with increased prevalence of mood disorders and offer a potential rational treatment approach. A similar relationship between omega-3 depletion and coronary artery disease may explain the well-described links between coronary artery disease and depression. Inconsistency in clinical trial results to date may reflect differences in the type and dose of omega-3 supplement chosen, the depression subtype and/or baseline omega-3 levels of the study participants.

\section{Meanings of religion and spirituality for adolescent out-patients}

\section{T Perich', M Dudley², DH Pavlovic' ${ }^{1}$, F Fitzjames ${ }^{2}$ \\ 'The Black Dog Institute, Sydney, Australia; and 2Prince of Wales Hospital,} Randwick, Australia

Spirituality is an important but often overlooked area in mental health. This study aimed to examine individual meanings regarding the concepts of religion and spirituality for adolescents attending a mental health out-patient service. Participants were 53 adolescent out-patients of the Department of Child and Adolescent Mental Health, Prince of Wales Hospital, Sydney, 
drawn from the early stage of larger study examining adolescent resilience. Adolescents were found to express eclectic and individual views surrounding the topic, with each response being uniquely representative of their beliefs. Themes commonly found included religion and God with many showing some degree of change. Some of these changes involved abandoning one religion in favor of another or changes in ideas about God. There was no significant relationship between those with a diagnosis and those without regarding their religious and spiritual beliefs; however, there were differences between groups in using beliefs to cope, beliefs about a spiritual force and its influence on day-to-day and world affairs. Spiritual and religious ideas are important for adolescents and those in the study showed a great diversity in ideas about this topic. It would be of further interest to research how these beliefs and ideas about religion and spiritual change over time and learn about how adolescents without a diagnosis use their beliefs to assist in coping.

\section{ECT and neuroprotection: a review and proposed study}

\section{Pigot, C Loo}

School of Psychiatry, University of New South Wales, Sydney, Australia

Background: Electroconvulsive therapy (ECT) is a highly effective treatment for depression but its use is limited by associated cognitive side-effects. Several theories have been proposed for the mechanisms underlying cognitive impairment with ECT: excitotoxic damage through influx of intracellular calcium or excessive glutamatergic transmission, alteration in opioid receptor density and induction of inflammatory processes (Krueger et al. 1992; Bazan et al. 2005). Several neuroprotective agents have been proposed to reduce ECT-related cognitive effects, leading to some trials in animal and human subjects.

Method: A comprehensive literature review (PubMed, Medline) identified animal and human clinical research trials of various agents proposed to have a neuroprotective effect on ECT-induced cognitive impairments.

Results: Several agents have been shown to reduce memory impairment after electroconvulsive shock (ECS) in rats, for example, calcium channel blockers, opioid receptor antagonists and glucocorticoid antagonists. However, results from clinical trials in humans have been less promising. There are early suggestions that ketamine anesthesia may be associated with fewer cognitive side-effects after ECT (McDaniels et al. 2006). Conclusions: Strong evidence from ECS models of neuroprotective strategies have to date not been confirmed in human clinical trials. However, clinical trials have been sparse, with small sample sizes and confounding methodological issues. We propose a randomized sham-controlled study to assess the effect of several neuroprotective agents on ECT-induced cognitive impairment.

\section{Spatial working memory task validation in a group of patients with schizophrenia and healthy volunteers}

\section{Piskulic ${ }^{1,2}, \mathrm{~J} \mathrm{Olver}^{1,2}, \mathrm{~K}$ Monshat ${ }^{2}$, T Norman $^{1,2}$, P Maruff ${ }^{3}$}

'The University of Melbourne, Melbourne, Australia; ${ }^{2}$ Austin Health; and

${ }^{3}$ CogState Ltd, Melbourne, Australia

Background: It is now agreed that cognitive deficit is an important aspect of the clinical presentation of schizophrenia. One parsimonious brain-behaviour model with good explanatory power for both animal and human models of the disorder is that of impairment of working memory (WM). Patients with schizophrenia show a reliable decrement of WM, which has been proposed to give rise to social and employment disabilities. The aim of the current study was to validate a computer-based behavioural spatial WM test in patients with schizophrenia.

Methods: A novel spatial WM test based on the ' $n$ back' paradigm was used to compare 15 medicated patients with schizophrenia against 15 healthy volunteers. The task involved remembering the position of a target card from an arrangement of between two and four cards presented in a radial configuration, with instructions to determine whether the card that was face up at the time of presentation was in the same location as a card that had turned face up on the previous trial (1-back condition) or two previous trials (2-back condition). All subjects were retested a week later to determine the stability and reliability of the test.

Results: Repeated-measures ANOVA showed that patients with schizophrenia performed worse than healthy controls on tests of spatial WM.

Conclusions: The behavioural $n$-back task proved to be a quick and reliable measure of spatial WM in patients with schizophrenia. Our data further support previous findings of spatial WM deficits in schizophrenia.

\section{Assessment of serotonin function, memory and spatial working memory using buspirone and placebo}

\footnotetext{
D Piskulic ${ }^{1,2}$, J Olver ${ }^{1,2}$, P Maruff ${ }^{3}$, T Norman ${ }^{1,2}$

'The University of Melbourne, Melbourne, Australia; ${ }^{2}$ Austin Health; and

${ }^{3}$ CogState Ltd, Melbourne, Australia
} 